\title{
The moderating role of negative affect on objective verbal memory performance and subjective memory complaints in healthy older adults
}

\author{
MOIRA C. DUX, ${ }^{1}$ JOHN L. WOODARD,${ }^{1}$ JOHN E. CALAMARI,${ }^{1}$ MICHAEL MESSINA, ${ }^{1}$ \\ SHALINI ARORA, ${ }^{1}$ HEATHER CHIK, ${ }^{1}$ AND NOELLE PONTARELLI ${ }^{1}$ \\ ${ }^{1}$ Department of Psychology, Rosalind Franklin University of Medicine and Science, North Chicago, Illinois \\ (Received May 1, 2007; Final Revision November 13, 2007; Accepted November 13, 2007)
}

\begin{abstract}
Subjective memory complaints (SMCs) are part of the diagnostic criteria for Mild Cognitive Impairment (MCI), yet little is known about their etiology. In some previous studies, no direct relation has been found between SMCs and objective memory performance, yet significant correlations have been identified between SMCs and psychological factors such as depression and anxiety. In the current study, we examined whether negative affect moderated the relation between objective memory functioning and SMCs in a sample of healthy, non-demented participants aged 65 and older. As predicted, several negative affect measures moderated the relationship between objective cognitive functioning and SMCs. In the absence of objective memory impairment as indexed by the Rey Auditory Verbal Learning Test (RAVLT) and the Dementia Rating Scale-2nd Edition (DRS-2), higher levels of negative affect were associated with increased levels of SMCs. Moreover, a lower order negative affect factor, anxiety sensitivity, significantly moderated the relation between objective memory functioning and SMCs, after controlling for higher order measures of general negative affectivity. Findings suggest that negative affect, particularly anxiety sensitivity, distorts the subjective appraisal of one's own memory, such that people high on negative affect factors report more episodes of forgetting, even in the absence of objective cognitive impairments. (JINS, 2008, 14, 327-336.)
\end{abstract}

Keywords: Aging, Anxiety, Depression, Self-efficacy, Mental recall, Retention (Psychology), Regression analysis

\section{INTRODUCTION}

Subjective memory complaints (SMCs) are common among older adults (Jungwirth et al., 2004), though their significance is not well understood. Recently, SMCs received greater clinical and research scrutiny in part because they are core diagnostic features of mild cognitive impairment (MCI; Petersen et al., 1999; Petersen et al., 2001). Although controversial, in a recent publication proposing the inclusion of MCI as a diagnostic entity in the Diagnostic and Statistical Manual of Mental Disorders (5th edition), Petersen and O'Brien (2006) retained the symptom "cognitive complaint" (modified from "subjective memory complaints"),

Correspondence and reprint requests to: John L. Woodard, Ph.D., Department of Psychology, Wayne State University, 5057 Woodward Ave., 7th Floor, Detroit, Michigan 48202. E-Mail: john.woodard@wayne.edu via self or informant report, as one of five core criteria for MCI.

Despite increased research attention to the nature of the subjective appraisal of cognitive functioning, there still is little agreement with respect to the etiology and clinical significance of SMCs. Presumably, SMCs would be related to objective memory performance. However, numerous cross-sectional studies failed to find significant relationships (e.g., Bolla et al., 1991; Jorm et al., 1994; Jungwirth et al., 2004; O'Connor et al., 1990; Schmidt et al., 2001; Sunderland et al., 1986), although a few exceptions do exist (Jonker et al., 1996; Lam et al., 2005; Podewils et al., 2003). Similar results emerge from longitudinal studies where a significant relationship between SMCs and objective memory performance is sometimes found (Dufouil et al., 2005; Guarch et al., 2004; Jorm et al., 2001; Martin \& Zimprich, 2003; Schmand et al., 1996; Wang et al., 2004), and some- 
times not (Frerichs \& Tuokko, 2006; Jorm et al., 1997; Taylor et al., 1992). Thus, there is little consensus regarding whether SMCs are associated with objective memory impairments or confer risk for future dementia.

Investigation of relationships between SMCs and brain pathology or genetic risk factors for dementia has also produced inconsistent findings. While some studies have failed to find an association between the presence of an apolipoprotein $\varepsilon 4$ allele (APOE $\varepsilon 4$ ) and SMCs in healthy older adults without objective evidence of a dementia (e.g., Clarnette et al., 2001; Harwood et al., 2004), other studies have found a significant relationship (e.g., Dik et al., 2001; Small et al., 1999; Stewart et al., 2001). These inconsistent findings may be attributed to psychological factors. Harwood et al. (2004) found a positive association between SMCs and depression in a sample of cognitively intact older adults, in the absence of an association between SMCs and APOE $\varepsilon 4$. Stewart et al. (2001) found that self-reported depressive symptoms moderated the association between SMCs and the APOE $\varepsilon 4$ allele, with the strongest relationship seen for participants meeting diagnostic criteria for Major Depressive Disorder. Still, some researchers have found a positive association between SMCs and white matter lesions in nondemented elderly subjects (Breteler et al., 1994; Saykin et al., 2006), not fully accounted for by depressive symptoms (de Groot et al., 2001). These findings suggest that SMCs could precede the onset of measurable deficits in objective cognitive functioning (de Groot et al., 2001). However, a study conducted by Minett et al. (2005) highlighted an association between SMCs and severity of white matter lesions, but found depression to be most strongly correlated with SMCs. Whereas the aforementioned studies provide somewhat disparate findings, it appears as if such inconsistencies may be partially explained by the influence of depressive symptoms.

In studies investigating alternative etiologies of SMCs, psychological factors appear to produce the most robust findings. In the absence of cognitive decline, Comijs et al. (2002) found a positive relationship between SMCs and physical health problems, depressive symptoms, anxiety symptoms and certain personality traits. Smith et al. (1996), found emotional status to be a better predictor of change in SMCs than current levels or changes in objective performance. Turvey et al. (2000) found depression and functional impairment to be predictors of SMCs in participants classified as being either "high" or "low" on cognitive performance. Additional studies have identified relationships between SMCs and anxiety, depression, psychosis and somatic symptoms (e.g., Clarnette et al., 2001; Grut et al., 1993; Hanninen et al., 1994; Harwood et al., 1998; Jorm et al., 1994; O'Connor et al., 1990). These studies suggest that psychological factors play an integral role in the development and perhaps the maintenance of SMCs.

Given the robust relationships between SMCs and psychological factors such as depression and anxiety, the current study aimed to explore the influence of general negative affect, a higher order factor encompassing both anxiety and depression (e.g., guilt, fear; Watson \& Pennebaker, 1989), and several lower-order negative affect variables (cf. Lilienfeld et al., 1993) on the relationship between SMCs and objective memory functioning. Healthy adults aged 65-years and older participated in the study.

Based on prior studies demonstrating a strong association between SMCs and negative affect factors such as depression and anxiety (e.g., Clarnette et al., 2001; Grut et al., 1993) and oftentimes a lack of a direct correlation between SMCs and objective memory functioning (e.g., Jorm et al., 1994; O'Connor et al., 1990), we predicted that negative affect would moderate the relationship between objective memory performance and SMCs. Older adults with high scores on general or specific measures of negative affect were predicted to report more SMCs and to be more reactive to even minor difficulties in cognitive functioning. That is, we predicted that older adults with higher negative affect would display increased SMCs even when performing at levels commensurate with age-based normative values.

\section{METHODS}

\section{Participants}

Data for this study were obtained from a larger, longitudinal study examining risk factors for late-life anxiety disorders in older adults. Subjects were primarily recruited from senior centers in Lake County, Illinois and Kenosha County, Wisconsin. Participants in the current study were 130 older adults $(M$ age $=76.7$ years, $S D=8.5$ Years; $71.8 \%$ female; $M$ education $=14.7$ years, $S D=2.8$ years; 94.7 white, $3.8 \%$ African American, .5\% Hispanic, $1.0 \%$ other). All participants evidencing objective cognitive impairment (Dementia Rating Scale-2 Total Raw Score $\leq 123$, DRS-2 Memory Subscale Scaled Score $<6$ and Rey Auditory Verbal Learning Test $z$ scores $<-1.30$ ) were excluded from the present analyses, therefore excluding participants with even mild deficits. All participants were administered the Structured Clinical Interview for DSM-IV Axis I Disorders, and individuals meeting criteria for a current or past disorder were excluded from the present analyses $(n=25)$.

\section{Negative Affect Measures}

\section{Geriatric Depression Scale (GDS; Yesavage et al., 1983):}

This self-report scale consists of 30 statements which require a "yes" or "no" response. The GDS focuses on the behavioral and cognitive aspects of depression while minimizing assessment of neurovegetative symptoms that frequently are related to causes other than depression (e.g., medical problems) in older adults. Several studies with various older adult samples (e.g., community, nursing home, medical inpatients) have found scores from the GDS to have good reliability and validity (e.g., Dunn \& Sacco, 1989; Koenig et al., 1988; Norris et al., 1987; Parmelee et al., 1989; Rapp et al., 
1988). Scores on this measure from our sample demonstrated good internal consistency $(\alpha=.91)$.

\section{Anxiety Sensitivity Index (ASI; Reiss et al., 1986):}

The ASI is a 16-item self-report measure of anxiety sensitivity (AS), which is defined as the fear of anxiety-related sensations due to a belief that these experiences will have harmful physical, social, or psychological consequences (Reiss \& McNally, 1985). AS is posited to be a lower order negative affect variable nested under trait anxiety (Lilienfeld et al., 1993). The participant reports the extent to which he/she agrees with each statement by circling one of five possible ratings ranging from very little to very much. Higher scores on this measure are thought to represent a risk factor for the development of anxiety disorders, particularly panic disorder. The ASI has demonstrated good psychometric properties including: test-retest reliability of $r=.71$ over a three year time interval in a large sample of college students (Maller \& Reiss, 1992), good predictive validity with respect to panic attacks and anxiety disorders (Reiss, 1991), and good internal consistency coefficients in large samples of college students $(\alpha=.82$, Telch et al., 1989; $\alpha=.88$, Peterson \& Heilbronner, 1987), as well as psychiatric outpatients ( $\alpha=.91$; Taylor et al., 1992). In our sample, scores from the ASI demonstrated good internal consistency $(\alpha=.87)$.

\section{Positive and negative affect schedule (PANAS; Watson et al., 1988):}

This instrument is a self-report measure consisting of 20 adjectives. Participants are asked to rate the extent to which they felt each adjective during the past week, ranging from $1=$ very slightly or not at all to $5=$ extremely. The measure consists of 10 positive affect adjectives (e.g., interested, excited) and 10 negative affect adjectives (e.g., irritable, distressed). Scores from the PANAS have very good internal consistency (PA $\alpha=.88$, NA $\alpha=.85$ ) and good convergent and discriminant validity among college students (Beck et al., 2003). In our sample, satisfactory internal consistency was found for both positive and negative affect factors: (NA) $\alpha=.85$, (PA) $\alpha=.91$.

\section{Penn State Worry Questionnaire (PSWQ; Meyer et al., 1990):}

The PSWQ is a 16-item self-report measure of worry. Items are rated from 1 -not typical at all to 5-very typical. Higher scores on this measure are indicative of increased worry. Scores from the PSWQ have shown high internal consistency, good test-retest reliability and construct validity in an adult sample with Generalized Anxiety Disorder (Meyer et al., 1990). The original form of the PSWQ contains several reverse-scored items that appear to be a problematic for older adults. Hopko et al. (2003) reduced the PSWQ from 16 to eight items, eliminating the five reverse scored items as well as the last three items on the scale. Crittendon \& Hopko (2006) evaluated the psychometric properties of this abbreviated Penn State Worry Questionnaire (PSWQ-A) among older adults and found strong internal consistency $(\alpha=.89)$, excellent test-retest reliability at two $(r=.92)$ and six weeks $(r=.95)$, moderate to strong convergent validity ( $r=.46-.83$ ) with measures of anxiety and worry, but weaker discriminant validity (correlation with Beck Depression Inventory-BDI, $r=.56$ ), compared to previous evaluations of the abbreviated PSWQ in older adults (correlation with BDI, $r=.16$, Hopko et al., 2003). This study employed the eight-item PSWQ because of its appropriateness for older adults. According to the method of McNemar (1950), a modified part-whole correlation was computed and found to be moderate $(r=.67)$. This relation is consistent with the findings of Crittendon and Hopko (2006), in which they also found a moderate relationship $(r=.65)$ between the eight item PSWQ and the full PSWQ, among older adults. In our sample, scores from the eight item PSWQ demonstrated good internal consistency $(\alpha=.89)$.

\section{Subjective Memory Complaints}

\section{Memory Functioning Questionnaire (MFQ) General Frequency of Forgetting (Gilewski et al., 1990):}

The MFQ is a self-report inventory that assess how people appraise their memory abilities. The full scale consists of four factors including: General Frequency of Forgetting, Seriousness of Forgetting, Retrospective Functioning and Mnemonics Usage. For the present study, only the General Frequency of Forgetting scale was used because it is most closely associated with current episodes of forgetting. Individuals are asked to rate how often certain information presents a problem with respect to remembering (e.g., faces, names, words, etc.) The internal consistency (Cronbach's alpha) of scores from this factor is .94 based on a sample ranging in age from 16-89 years (Gilewski et al., 1990). It is important to note that lower scores on this measure are indicative of increased subjective memory complaints. In our sample, scores on this factor demonstrated good internal consistency $(\alpha=.95)$.

\section{Objective Memory/Cognitive Functioning}

\section{Dementia Rating Scale-2 (DRS-2; Jurica et al., 2001, Mattis, 1988).}

The DRS-2 is a screen for cognitive impairment that consists of five subscales: attention, initiation/perseveration, construction, conceptualization, and memory. Scores from the DRS-2 exhibit good test-retest reliability (one week interval) and internal consistency (Jurica et al., 2001). Moderate to strong correlations with other measures, such as the Wechsler Adult Intelligence Scale-Revised and Wechsler Mem- 
ory Scale, suggest good convergent and discriminant validity in healthy and cognitively impaired older adults (Jurica et al., 2001). For the purposes of the current study, the total raw score was used as an index of general cognitive functioning. Because objective memory functioning was also crucial to our central hypotheses, we used the age-scaled score from the Memory Subscale score of the DRS-2 as the index of objective memory functioning to minimize the impact of age-related memory changes. Age-scaled scores were based on the Mayo Older American Normative Studies (Lucas et al., 1998) and range from 2-18, whereas the total raw score on the DRS-2 can range from 0-144.

\section{Rey-Auditory Verbal Learning Test (Rey, 1964).}

The AVLT is a verbal list learning task that indexes immediate memory, learning over trials, and retention following a 20 minute delay. This task consists of 5 immediate presentations of a 15-item word list and one delayed recall trial. Scores from the AVLT have demonstrated relatively good test-retest reliability and good concurrent validity. Moreover, the AVLT has been frequently used in both younger and older adults and is sensitive to memory impairment (Schmidt, 1996). Age-based $z$ scores were calculated for total immediate recall (sum of trials 1-5) and delayed recall.

\section{Procedure}

All participants provided informed consent. self-report measures, along with detailed instructions for proper completion, were mailed to participants approximately two weeks prior to their cognitive/psychological evaluation. The cognitive and psychological testing was administered by advanced clinical psychology doctoral students extensively trained in test administration and scoring by two licensed clinical psychologists. Subjects were provided a \$25 honorarium for their participation. Each participant's results were reviewed by two licensed clinical psychologists for accuracy of administration and interpretation of findings. This study was fully approved by the Institutional Review Board at Rosalind Franklin University of Medicine and Science.

\section{Data Analysis}

All data were analyzed using SPSS version 14 (SPSS, Inc., Chicago, Illinois, 2005). Regression diagnostics were used to assess homoscedasticity of residuals, outliers and multicollinearity. Outliers greater than three standard deviations were excluded from the analyses ( $n=4$ cases). Tolerance levels below .10 and variance inflation factors greater than 10 are generally thought to be indicative of multicollinearity (Cohen et al., 2003). All tolerance values were greater than .75 and all variance inflation factors were less than 2 .

In order to determine the relationship between subjective and objective cognitive performance, several moderator models were tested using regression analyses (e.g., Aiken \& West, 1991; Jaccard et al., 1990). For all models, subjective memory functioning served as the dependent variable, indexes of objective memory performance as the independent variable, and negative affect variables as the moderator. As suggested by (Jaccard et al., 1990) predictor variables were mean centered and significant interactions were probed at $+/-1$ standard deviation from the mean score reported by the sample. SPSS syntax written by O'Connor (1998) was used to test and probe interactions.

\section{RESULTS}

Descriptive statistics and simple correlations for all variables are presented in Table 1. All negative affect measure means were within the nonclinical range and cognitive functioning measures were within normal limits compared to age-adjusted data. Participants with scores on any measure greater than three standard deviations from the mean were labeled as outliers and excluded form all analyses $(n=10)$. All participants with either total raw scores on the DRS- $2<123$, Memory Subscale scaled scores of $<6$

Table 1. Descriptive and correlation data for cognitive, negative affect, and SMCS variables

\begin{tabular}{|c|c|c|c|c|c|c|c|c|c|c|c|}
\hline Variables & $M$ & $S D$ & 1 & 2 & 3 & 4 & 5 & 6 & 7 & 8 & 9 \\
\hline 1. MFQ Freq & 167.6 & 27.4 & & & & & & & & & \\
\hline 2. DRS Total & 138.4 & 4.9 & $.30 * *$ & & & & & & & & \\
\hline 3. DRS Mem & 11.0 & 2.4 & $.24 * *$ & $.67 * *$ & & & & & & & \\
\hline 4. ASI & 15.5 & 9.4 & $-.44 * *$ & -.02 & -.11 & & & & & & \\
\hline 5. GDS & 6.0 & 6.0 & $-.35 * *$ & .07 & .05 & $.41 * *$ & & & & & \\
\hline 6. NA & 14.4 & 5.2 & $-.24 * *$ & .16 & .07 & $.21 *$ & $.66 * *$ & & & & \\
\hline 7. PSWQ & 15.5 & 7.2 & $-.37 * *$ & .00 & -.03 & $.46^{* *}$ & $.59 * *$ & $.46 * *$ & & & \\
\hline 8. AVLT-IR & .64 & 1.3 & $.30 * *$ & $.52 * *$ & $.32 * *$ & -.01 & .05 & .02 & .08 & & \\
\hline 9. AVLT-DR & .41 & 1.3 & $.24 * *$ & $.28 * *$ & $.19^{*}$ & -.04 & .04 & -.02 & .02 & $.80 * *$ & \\
\hline
\end{tabular}

Note. MFQ Freq = Memory Functioning Questionnaire, Frequency Scale; DRS Total = Dementia Rating Scale—2 Total Raw Score; DRS Mem = Dementia Rating Scale-2, Memory Scale; ASI = Anxiety Sensitivity Index; GDS = Geriatric Depression Scale; NA = negative affect score from Positive and Negative Affect Schedule, PA= positive affect score from Positive and Negative Affect Schedule; PSWQ = Penn State Worry Questionnaire—8 item; AVLT-IR = Auditory Verbal Learning Test—Immediate Recall; AVLT$\mathrm{DR}=$ Auditory Verbal Learning Test-Delayed Recall. $* p<.05 . * * p<.001$. 
on the DRS-2, or immediate recall or delayed recall $\mathrm{z}$ scores from the AVLT $<-1.30$ were excluded from analyses $(n=19)$.

Correlational analyses were conducted to determine the relation between objective memory performance, negative affect and SMCs. The MFQ frequency scale was positively correlated with the DRS-2 total raw score, the DRS-2 Memory Subscale and the AVLT-immediate recall and delayed recall, indicating that better cognitive functioning was associated with fewer SMCs. In addition, all negative affect measures were negatively correlated with the MFQ frequency of forgetting scale, indicating that as negative affect scores increased, SMCs also increased. Again, lower scores on the MFQ are indicative of increased SMCs. All negative affect variables were significantly correlated with one another as expected.

\section{Moderated Regression Analyses}

Based on our a priori hypotheses that negative affect measures would moderate the relation between SMCs and objective cognitive/memory functioning, we analyzed several moderator models. First, we tested the moderator model with general negative affect (i.e., PANAS negative affect) and then for each lower-order anxiety or depression measure. For each independent model in the initial set of analyses, the DRS-2 age-corrected Memory Subscale score served as the independent variable, the selected negative affect measure as the moderator and the MFQ Frequency of Forgetting factor as the dependent variable. All moderator models were significant and probing each of the interactions at $+/-1$ standard deviations from the mean, revealed significant simple slopes at medium and high levels of each of the specified negative affect moderators. The results of each of these models are presented in Table 2.

\section{The Relation of Anxiety Sensitivity to SMCs, Controlling for Higher Order Negative Affectivity}

Among the lower order negative affect variables examined in this study, all were moderately correlated with the higher order general negative affectivity measure (PANAS, NA) at $p<.001$ (GDS, $r=.66$, PSWQ, $r=.46$ ), with the exception of anxiety sensitivity, which was only weakly correlated $(r=.21, p<.05)$. Thus, it appeared that the ASI was measuring unique aspects of psychological distress not solely accounted for by general negative affectivity. Therefore, we examined the incremental validity of the Anxiety Sensitivity Index in moderating the relationship between objective memory performance across two separate measures of memory functioning and SMCs. In the initial set of sequential regression analyses, we controlled for general negative affectivity, depression and worry (PANAS, NA; GDS; PSWQ) and looked at the interaction between the ASI and the Memory Subscale from the DRS-2, in predicting SMCs. The overall interaction term was significant $\left(F=8.37, p=.005, R^{2} \Delta=.059\right)$ and probing of the interaction revealed significant medium $(M: \beta=.201, p=.019)$ and high $(M+1 S D: \beta=.471$, $p<.001)$ simple slopes. Therefore, even after controlling for higher order negative affectivity factors, increases on anxiety sensitivity were associated with increased SMCs in the absence of objective memory impairments (Fig. 1). Moreover, when we tested the converse question, that is, whether higher order negative affectivity (PANAS, NA; GDS; PSWQ) moderated the relationship between objective memory performance and SMCs after controlling for the influence of anxiety sensitivity, the overall interaction was not significant $\left(F=2.24, p=.137, R^{2} \Delta=.016\right)$, further demonstrating the unique effects of anxiety sensitivity on this relationship.

In the second set of analyses, we aimed to determine whether anxiety sensitivity, after controlling for higher order negative affect factors (PANAS-NA, GDS, PSWQ), interacted with a more challenging list-learning memory task to predict SMCs. For the first moderator model, age-based $z$ scores for total immediate recall (sum of Trials 1-5) from the AVLT were employed as the independent variable, ASI as the moderator and the MFQ Frequency of Forgetting factor as the dependent variable. The overall interaction term was significant $\left(F=4.07, p=.046 ; R^{2} \Delta=.028\right)$. Probing of the interaction revealed significant simple slopes at medium $(M: \beta=.265, p=.002)$ and high $(M+1 S D$ : $\beta=.441, p=.001)$ levels of the moderator. Thus, irrespective of the influence of higher order negative affect factors, anxiety sensitivity uniquely interacted with auditory verbal learning to predict SMCs, such that increased scores on the

Table 2. Moderated regression statistics: DRS memory subscale $\times$ NA mod $=$ MFQ freq DV

\begin{tabular}{lcccccccr}
\hline \hline Moderator & $N$ & $d f$ & $R^{2} \Delta$ & $F$ & $P$ & Low $\beta$ & Med $\beta$ & High $\beta$ \\
\hline NA & 130 & 3 & .046 & 7.10 & .009 & .026 & $.288^{* *}$ & $.551^{* *}$ \\
PSWQ & 130 & 3 & .056 & 9.37 & .003 & .002 & $.250^{* *}$ & $.499 * *$ \\
GDS & 130 & 3 & .084 & 14.64 & .000 & -.033 & $.292^{* *}$ & $.618^{* *}$ \\
ASI & 130 & 3 & .048 & 8.36 & .005 & -.063 & $.181^{*}$ & $.424 * *$ \\
\hline \hline
\end{tabular}

Note. NA Mod = Negative Affect Moderator; NA = negative affect score from Positive and Negative Affect Schedule (PANAS); PSWQ = Penn State Worry Questionnaire-8 item; GDS = Geriatric Depression Scale; ASI $=$ Anxiety Sensitivity Index. 


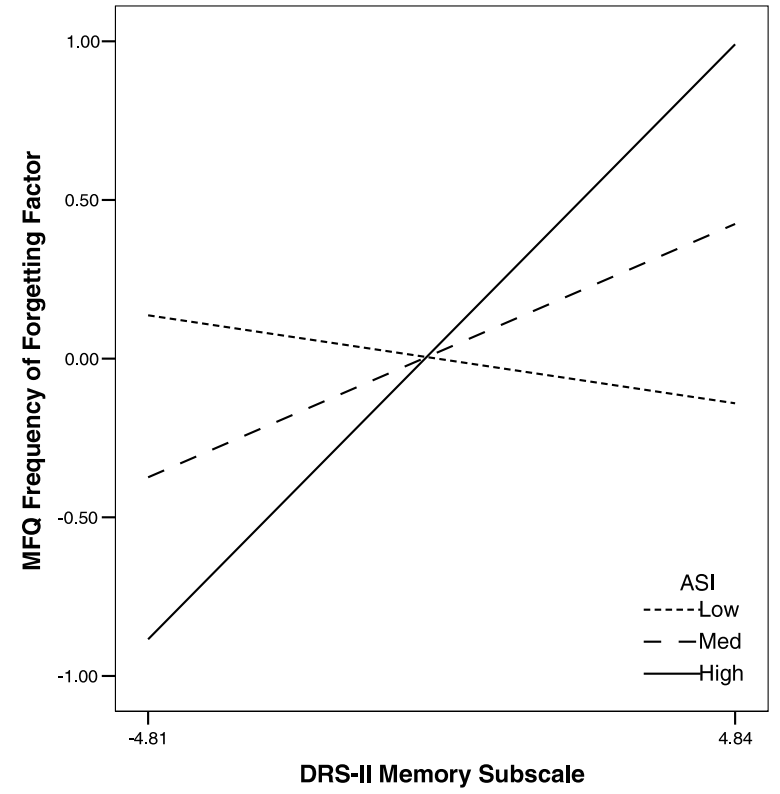

Note. ASI = Anxiety Sensitivity Index; MFQ Frequency of Forgetting-Memory Functioning Questionnaire-Frequency of Forgetting factor; NA = Negative Affect; Low $=M-1 S D$, Medium $=M, \operatorname{High}=M+1 S D$.

Fig. 1. DRS-2 Memory Scale X ASI = MFQ Freq (controlling for higher order NA variables)

ASI were associated with increased SMCs, in the absence of objective impairments in one's ability to learn words over immediate recall trials of a 15 -item word list.

In the final set of moderated regression analyses, we aimed to determine whether the ASI interacted with delayed

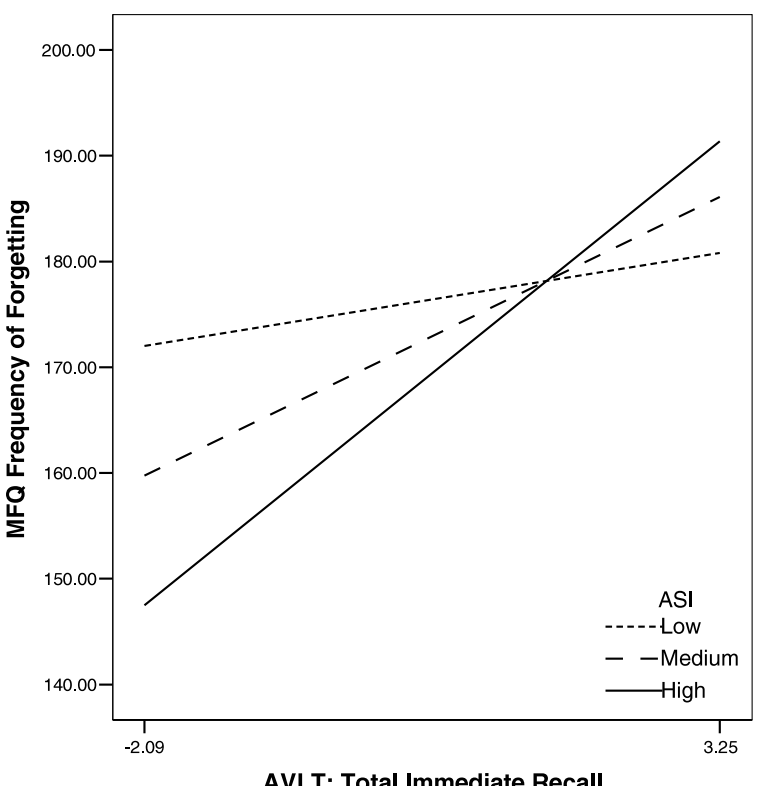

Note. ASI = Anxiety Sensitivity Index; MFQ Frequency of Forgetting-Memory Functioning Questionnaire-Frequency of Forgetting factor; AVLT-Total Immediate Memory —Auditory Verbal Learning Test—Total Immediate Recall; NA = Negative Affect; Low $=M-1 S D$, Medium $=M, \operatorname{High}=M+1 S D$.

Fig. 2. AVLT Total Immediate Recall X ASI $=$ MFQ Freq (controlling for higher order NA variables)

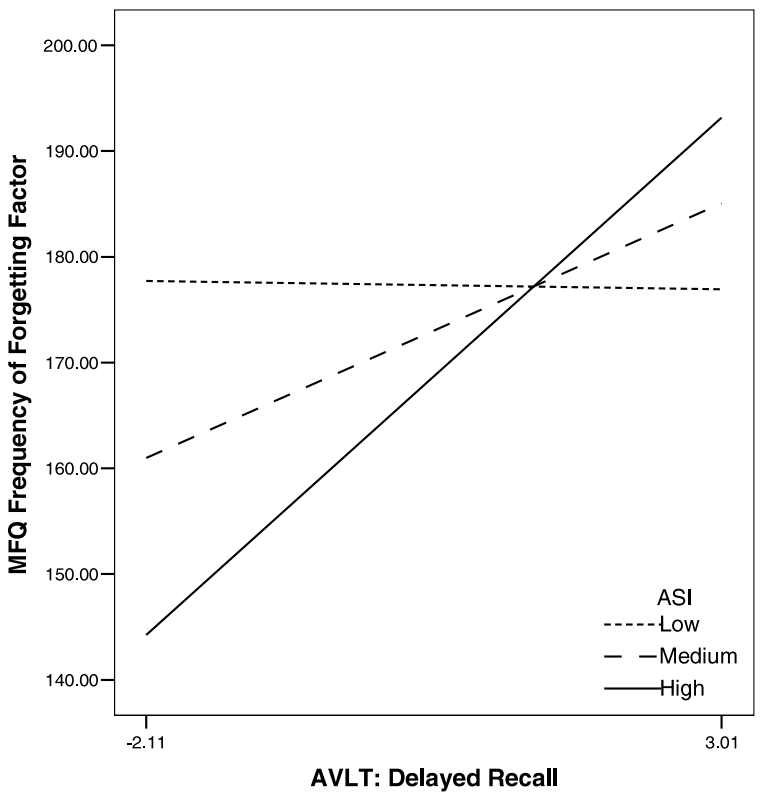

Note. ASI = Anxiety Sensitivity Index; MFQ Frequency of Forgetting-Memory Functioning Questionnaire-Frequency of Forgetting factor; AVLT-Total Delayed Recall-Auditory Verbal Learning Test-Delayed Recall; NA = Negative Affect; Low $=M-1 S D$, Medium $=M$, High $=M+1 S D$.

Fig. 3. AVLT Total Delayed Recall X ASI $=$ MFQ Freq (controlling for higher order NA variables)

recall on the AVLT to predict SMCs, after controlling for higher order negative affect factors (PANAS-NA, GDS, PSWQ). The overall regression equation was significant $\left(F=8.16, p=.005, R^{2} \Delta=.059\right)$ and probing of the interaction revealed significant medium $(M: \beta=.245, p=$ $.004)$ and high $(M+1 S D: \beta=.504, p<.001)$ simple slopes. Therefore, as one's anxiety sensitivity, or the fear of experiencing anxiety symptoms increases, so does one's frequency of SMCs, despite intact performance on a delayed recall trial of a 15 -item list learning task.

\section{DISCUSSION}

In this study, we aimed to examine the relationship among negative affect, SMCs and objective memory functioning in a non-demented elderly sample. The findings demonstrate the influence of several aspects of negative affect, particularly anxiety sensitivity, on the relation between objective cognitive functioning and SMCs. In continuous by continuous moderated regression analyses, we tested four independent measures of negative affect, conceptualized as representing different levels of a hierarchical orders of negative affect. Specifically, in accordance with Lilienfeld et al. (1993), we conceptualized the PANAS NA score as representing a higher order, trait-like assessment of general negative affectivity, the PSWQ and the GDS as representing second order, state measures of negative affectivity, and fear of anxiety, as assessed by the ASI, as representing a lower order negative affect factor. Scores from the ASI, 
GDS, PANAS-NA, and the PSWQ (8-item) were all significant independent moderators of the relationship between objective memory performance (DRS-2 memory subscale) and SMCs (MFQ Frequency of Forgetting scale). Simple slope analyses revealed that for each of the four models, at medium $(M)$ and high $(M+1 S D)$ levels of negative affect, increased scores on each measure were associated with greater perceived frequency of forgetting for a given level of objective cognitive functioning. These findings suggest that several negative affect factors may distort the subjective appraisal of one's own memory functioning, causing one to believe that he/she is forgetting information more frequently than is actually the case.

Anxiety sensitivity has been conceptualized as a "fear of anxiety" and has been shown to be a risk factor for the development of certain types of anxiety disorders, particularly panic disorder (e.g., Kotov et al., in press; Schmidt et al., 1998). Based on Lilienfeld et al. (1993), anxiety sensitivity can be conceptualized as a lower order negative affect variable, and thus, the most distinct variable included in the present study from the higher order, general trait negative affectivity measure, PANAS NA. In order to evaluate the incremental validity of anxiety sensitivity in moderating the relation between objective memory performance and SMCs, we re-examined the relationship between DRS-2 Memory Subscale and SMCs, controlling for all other negative affect factors (PANAS, NA, GDS, PSWQ). Again, the interaction was significant and further probing revealed that increasing levels of anxiety sensitivity were associated with increased SMCs, in the absence of impaired objective cognitive functioning.

To examine whether our findings generalized to a more challenging memory measure, we examined the interaction between anxiety sensitivity (after controlling for other negative affect variables), and different indicators of performance on the AVLT in predicting SMCs. Indeed, we found that increased anxiety sensitivity was associated with increased SMCs, in the absence of objective memory impairments. Thus, the interaction between anxiety sensitivity and several indices of objective memory functioning (e.g., DRS memory subscale, AVLT immediate \& delayed recall) in predicting SMCs, appears to be quite robust. Our results suggest that anxiety sensitivity, or a fear of anxiety related symptoms, is associated with distorted appraisal of one's own memory, such that increased episodes of forgetting are reported in the absence of underlying objective memory deficits.

Based on our data, it seems as though negative affectivity, particularly anxiety sensitivity, distorts subjective appraisal of one's memory. However, there are several plausible mechanisms, other than faulty appraisals, that should be entertained. Whereas there is some debate regarding the ability to reliably differentiate between dementia and depression (for review see: Amore et al., 2007), depression is the most common psychiatric comorbidity associated with Alzheimer's disease and is typically part of the early symptom presentation of dementia. In addition, neuropsychiatric symptoms, most commonly depression, are overrepresented in pre-clinical dementia states such as mild cognitive impairment (Lyketsos et al., 2002). Thus, it is possible that increases in negative affect, primarily depression, may precede obvious cognitive deficits, as indexed by neuropsychological measures. Additionally, the "vascular depression" hypothesis, which posits that vascular disease puts one at risk for, precedes or advances depression (Thomas et al., 2004) would argue that changes in cognition and affect share underlying neurological mechanisms, such as white matter signal hyperintensities. Thus, it is possible that increased negative affect, primarily depression, may be indicative of decrements in cognitive functioning. It is unlikely however, that the influence of depression and its complex ties with cognitive changes in late-life principally account for our findings. All participants meeting criteria for a past or present Axis I disorder were excluded and all participants included were far below clinical cut-offs with respect to scores on selfreport measures of depression. Last, whereas depression was found to be a significant moderator of the relationship between objective memory functioning and SMCs, anxiety sensitivity, was the only moderator that remained significant after controlling for the effects of other negative affect variables, including depression.

Although the relationship between objective memory functioning, SMCs and different ordered factors of negative affectivity has not previously been assessed, several studies have documented a positive correlation between negative affectivity and physical symptom reporting (Koller et al., 1996; Stegen et al., 2001; Watson \& Pennebaker, 1989). Our study extends these findings in that we found a significant relationship between all negative affect measures and our measure of SMCs. In other words, increased self-reports of negative affect were associated with increased perceived episodes of forgetting. In order to place our findings into a theoretical context, we examined the relevance of the symptom perception hypothesis (Watson, 1988; Watson \& Pennebaker, 1989), to our data. This hypothesis posits that individuals with high negative affect vary in the manner in which they view, respond and complain about bodily sensations. The most extreme form of this model claims that the relationship between negative affect and health complaints is spurious and simply demonstrates that individuals with high negative affect have an increased tendency to attend to and complain about internal somatic sensations. Based on our findings, we propose an extension of the symptom perception hypothesis to include not only perception of physical problems, but appraisal of cognitive functioning as well. In the absence of objective memory impairments, we found that increased negative affect, primarily anxiety sensitivity, was associated with increased SMCs. Based on our data, one could argue that individuals with high negative affect, particularly anxiety sensitivity, have an increased tendency to attend to and complain about cognitive functioning, even when no evidence of objective cognitive impairments exist.

It is important to note several limitations of this study that restrict the generalizability of the findings. First and 
foremost, this study was correlational and cross-sectional in nature, so we are unable to evaluate causal mechanisms underlying the relation between SMCs, objective memory functions and negative affect. Moreover, with the current data, we are unable to evaluate how changes over time in either objective memory functioning, self-reported levels of negative affect or SMCs affect the relationship among such variables. It is also possible that some individuals in our sample experienced a relative decline in their cognitive abilities, were cognizant of such declines and thus reported increased negative affect and SMCs. Given the crosssectional nature of our data, we are unable to evaluate this alternative explanation, but we are currently collecting longitudinal data to address such questions. Moreover, our sample was predominately female and white, thus restricting the generalizability of our findings beyond these demographic characteristics. Additionally, our findings only generalize to individuals who do not evidence objective cognitive impairments and score within normal limits on self-report measures of affectivity. Thus, our results cannot be extended to those meeting criteria for diagnoses of affective disorders or cognitive decline beyond the normal level for a given age. However, it is possible that the relationship between clinically significant affective distress, objective memory functioning, and SMCs may be even more robust than that observed in our healthy sample, thus posing an exciting direction for future research. Lastly, the measures used in this study to assess verbal memory functioning (DRS-2, AVLT), do not adequately assess non-verbal memory abilities. Thus, it is possible that those performing in the normal range on verbal memory measures may have evidenced impairments on nonverbal memory measures, if assessed.

Despite these limitations, this study lends further evidence to the growing body of literature demonstrating a relationship between psychological factors and SMCs (e.g., Clarnette et al., 2001; Hanninen et al., 1994). To our knowledge, this study is the first to examine the relationships of broader psychological constructs, such as negative affect, to SMCs. This study adds further support for the role of psychological variables as potential explanatory factors for the inconsistent findings between SMCs and objective memory performance. Although our study was conducted on a healthy sample of older adults with a wide range of memory abilities, our results underscore the importance of routinely integrating cognitive and affective assessment measures into assessment of individuals with possible MCI. This study suggests that the presence of self-reported memory complaints does not always accurately reflect the degree of objective memory functioning. We are not arguing however that "cognitive complaints" should be removed as part of diagnostic criteria for MCI, instead we are suggesting they be interpreted within an affective context. Again, we suggest that clinicians as well as those overseeing community screening programs, include psychological assessments, particularly of negative affectivity, into cognitive batteries. Fortunately, several affective measures, well- validated in elderly populations (e.g., GDS) are quite succinct and thus should not significantly lengthen clinical evaluations. Moreover, based on our data, we feel that inclusion of a measure of anxiety sensitivity is warranted given the robust effect of anxiety sensitivity on the relationship between objective memory functioning and SMCs. In summary, this study demonstrates the robust influence of negative affect on relationships between multiple indicators of objective memory functioning and SMCs in which greater negative affect, particularly anxiety sensitivity, is associated with increased perceived episodes of forgetting in the absence of objective memory impairments.

\section{ACKNOWLEDGMENTS}

This research was supported by grant R21 MH069704 from the National Institute of Mental Health. There are no additional financial or other relationships that could be interpreted as a conflict of interest affecting this manuscript. Portions of this manuscript were presented at the annual meeting of the International Neuropsychological Society, Portland, Oregon, February, 2007. This study represents a portion of Moira Dux's thesis submitted in partial fulfillment of the degree of Master of Science at Rosalind Franklin University of Medicine and Science, performed under the supervision of John L. Woodard and John E. Calamari. John L. Woodard is now in the Department of Psychology at Wayne State University

\section{REFERENCES}

Aiken, L.S. \& West, S.G. (1991). Multiple regression: Testing and interpreting interactions. Thousand Oaks, CA: Sage.

Amore, M., Tagariello, P., Laterza, C., \& Savoia, E.M. (2007). Subtypes of depression in dementia. Archives of Gerontology and Geriatrics, Suppl. 1, 23-33.

Beck, J.G., Novy, D.M., Diefenbach, G.J., Stanley, M.A., Averill, P.M., \& Swann, A.C. (2003). Differentiating anxiety and depression in older adults with generalized anxiety disorder. Psychological Assessment, 15, 184-192.

Bolla, K.I., Lindgren, K.N., Bonaccorsy, C., \& Bleeker, M.L. (1991). Memory complaints in older adults. Archives of Neurology, 48, 61-64.

Breteler, M.M.B., van Sweiten, J.C., Bots, M.L., Grobbee, D.E., Claus, J.J., van den Hout, J.H.W., van Harskamp, F., Tanghe, H.L.J., de Jong, P.T.V.M., van Gijn, J., \& Hofman, A. (1994). Cerebral white matter lesions, vascular risk factors, and cognitive function in a population-based study: The Rotterdam Study. Neurology, 44, 1246-1252.

Clarnette, R.M., Almeida, O.P., Forstil, H., Paton, A., \& Martins, R.N. (2001). Clinica characteristics of individuals with subjective memory loss in Western Australia: Results from a crosssectional survey. International Journal of Geriatric Psychiatry, 16, 168-174.

Cohen, J., Cohen, P., West, S.G., \& Aiken, L.S. (2003). Applied multiple regression/correlation analysis for the behavioral sciences (3rd ed.). Mahwah, NJ: Lawrence Erlbaum Associates, Publishers.

Comijs, H.C., Deeg, D.J.H., Dik, M.G., Twisk, J.W.R., \& Jonker, C. (2002). Memory complaints; the association with psychoaffective and health problems and the role of personality characteristics: A 6 year follow-up study. Journal of Affective Disorders, 72, 157-165. 
Crittendon, J. \& Hopko, D.R. (2006). Assessing worry in older and younger adults: Psychometric properties of an abbreviated Penn State Worry Questionnaire (PSWQ-A). Journal of Anxiety Disorders, 20, 1036-1054.

de Groot, J.C., de Leeuw, F.E., Oudkerk, M., Hofman, A., Jolles, J., \& Breteler, M.M.B. (2001). Neurology, 56, 1539-1545.

Dik, M.G., Jonker, C., Comijis, H.C., Bouter, L.M., Twisk, J.W.R., van Kamp, G.J., \& Deeg, D.J.H. (2001). Memory complaints and APOE-4 accelerate cognitive decline in cognitively normal elderly. Neurology, 57, 2217-2222.

Dufouil, C., Fuhrer, R., \& Alperovitch, A. (2005). Subjective cognitive complaints and cognitive decline: Consequence or predictor? The epidemiology of Vascular Aging Study. Journal of the American Geriatrics Society, 53, 616-621.

Dunn, V.K. \& Sacco, W.P. (1989). A psychometric evaluation of the Geriatric Depression Scale and the Zung Self-Rating Depression Scale using an elderly community sample. Psychology and Aging, 4, 125-126.

Frerichs, R.J. \& Tuokko, H.A. (2006). Reliable change scores and their relation to perceived change in memory: Implications for the diagnosis of mild cognitive impairment. Archives of Clinical Neuropsychology, 21, 109-115.

Gilewski, M.J., Zelinski, E.M., \& Schaie, K.W. (1990). The Memory Functioning Questionnaire for assessment of memory complaints in adulthood and old age. Psychology and Aging, 5, 482-490.

Grut, M., Jorm, A.F., Fratiglioni, L., Forsell, Y., Viitanen, M., \& Winblad, B. (1993). Memory complaints of elderly people in a population survey: Variation according to dementia stage and depression. Journal of the American Geriatrics Society, 41, 1295-1300.

Guarch, J., Marcos, T., Salamero, M., \& Blesa, R. (2004). Neuropsychological markers of dementia in patients with memory complaints. International Journal of Geriatric Psychiatry, 19, 352-358.

Hanninen, T., Reinikainen, K.J., Helkala, E., Koivisto, K., Mykkänen, L., Laakso, M., Pyörälä, K., \& Riekkinen, P.J. (1994). Subjective memory complaints and personality traits in normal elderly subjects. Journal of the American Geriatrics Society, 42, 1-4.

Harwood, D.G., Barker, W.W., Ownby, R.L., \& Duara, R. (1998). Memory complaints in the elderly: A comparative analysis of informant and subjects reports among Hispanics and with NonHispanics. Clinical Gerontologist, 18, 56-60.

Harwood, D.G., Barker, W.W., Ownby, R.L., Mullan, M., \& Duara, R. (2004). No association between subjective memory complaints and apolipoprotein E genotype in cognitive intact elderly. International Journal of Geriatric Psychiatry, 19, 1131-1139.

Hopko, D.R., Stanley, M.A., Reas, D.L., Wetherell, J.L., Beck, J.G., Novy, D.M., \& Averill, P.M. (2003). Assessing worry in older adults: Confirmatory factor analyses of the Penn State Worry Questionnaire and psychometric properties of an abbreviated model. Psychological Assessment, 15, 173-183.

Jaccard, J., Wan, C.K., \& Turrisi, R. (1990). The detection and interpretation of interaction effects between continuous variables in multiple regression. Multivariate Behavioral Research, $25,467-478$.

Jonker, C., Launer, L.J., Hooijer, C., \& Lindeboom, J. (1996). Memory complaints and memory impairment in older individuals. Journal of the American Geriatrics Society, 44, 44-49.

Jorm, A.F., Christensen, H., Henderson, A.S., Korten, A.E., Mackinnon, A.J., \& Scott, R. (1994). Complaints of cognitive decline in the elderly: A comparison of reports by subjects and infor- mants in a community survey. Psychological Medicine, 24, 365-374.

Jorm, A.F., Christensen, H., Korten, A.E., Henderson, A.D., Jacomb, P.A., \& Mackinnon, A. (1997). Do cognitive complaints predict future cognitive decline or reflect past cognitive decline? A longitudinal study of an elderly community sample. Psychological Medicine, 27, 91-98.

Jorm, A.F., Christensen, H., Korten, A.E., Jacomb, P.A., \& Henderson, A.S. (2001). Memory complaints as a precursor of memory impairment in older people: A longitudinal analysis over 7-8 years. Psychological Medicine, 31, 441-449.

Jungwirth, S., Fishcer, P., Weissgram, S., Kirchmeyr, W., Bauer, P., \& Tragl, K.H. (2004). Subjective memory complaints and objective memory impairment in the Vienna-Transdanube aging community. Journal of the American Geriatrics Society, 52, 263-268.

Jurica, P.J., Leitten, C.L., \& Mattis, S. (2001). Dementia Rating Scale-2: Professional manual. Lutz, FL: Psychological Assessment Resources.

Koenig, H.C., Meador, K.G., Cohen, H.J., \& Blazer, D.G. (1988). Self-rated depression scales and screening for major depression in the older hospitalized patient with medical illness. Journal of the American Geriatrics Society, 36, 699-706.

Koller, M., Kussman, J., Lorenz, W., Jenkins, M., Voss, M., Arens, E., Richter, E., \& Rothmund, M. (1996). Symptom reporting in cancer patients. Cancer, 77, 983-995.

Kotov, R., Watson, D., Robles, J.P., \& Schmidt, N.B. (in press). Personality traits and anxiety symptoms: The multilevel trait predictor model. Behavior Research \& Therapy.

Lam, L.C.W., Lui, V.W.C., Tam, C.W.C., \& Chiu, H.F.K. (2005). Subjective memory complaints in Chinese subjects with mild cognitive impairment and early Alzheimer's disease. International Journal of Geriatric Psychiatry, 20, 876-882.

Lilienfeld, S.O., Turner, S.M., \& Jacob, R.G. (1993). Anxiety sensitivity: An examination of theoretical and methodological issues. Advances in Behaviour Research and Therapy, 15, 147-183.

Lucas, J.A., Ivnik, R.J., Smith, G.E., \& Bohac, D.L. (1998). Normative data for the Mattis Dementia Rating Scale. Archives of Clinical Neuropsychology, 13, 41-42.

Lyketsos, C.G., Lopez, O., Jones, B., Fitzpatrick, A.L., Breitner, J., \& DeKofsky, S. (2002). Prevalence of neuropsychiatric symptoms in dementia and mild cognitive impairment. Journal of the American Medical Association, 288, 1475-1483.

Maller, R.G. \& Reiss, S. (1992). Anxiety sensitivity in 1984 and panic attacks in 1987. Journal of Anxiety Disorders, 6, 241-247.

Martin, M. \& Zimprich, D. (2003). Are changes in cognitive functioning in older adults related to changes in subjective complaints? Experimental Aging Research, 29, 335-352.

Mattis, S. (1988). Dementia Rating Scale Professional Manual. Odessa, FL: Psychological Assessment Resources.

McNemar, Q. (1950). On abbreviated Wechsler-Bellevue scales. Journal of Consulting Psychology, 14, 79-81.

Meyer, T.J., Miller, M.L., Metzger, R.L., \& Borkovec, T.D. (1990). Development and validation of the Penn State Worry Questionnaire. Behavior Research \& Therapy, 28, 487-495.

Minett, T.S.C., Dean, J.L., Firbank, M., English, P., \& O’Brien, J.T. (2005). Subjective memory complaints, white-matter lesions, depressive symptoms, and cognition in elderly patients. American Journal of Geriatric Psychiatry, 13, 665-671.

Norris, J.T., Gallagher, D., Wilson, A., \& Winograd, C.H. (1987). Assessment of depression in geriatric medical outpatients: The 
validity of two screening measures. Journal of the American Geriatrics Society, 35, 989-995.

O'Connor, B.P. (1998). All-in-one programs for exploring interactions in moderated multiple regression. Educational and Psychological Measurement, 58, 833-837.

O'Connor, D.W., Pollitt, P.A., Roth, M., Brook, P.B., \& Reiss, B.B. (1990). Memory complaints and impairment in normal, depressed, and demented elderly persons identified in a community survey. Archives of General Psychiatry, 47, 224-227.

Parmelee, P.A., Lawton, M.P., \& Katz, I.R. (1989). Psychometric properties of the Geriatric Depression Scale among the institutionalized aged. Psychological Assessment: A Journal of Consulting and Clinical Psychology, 1, 331-338.

Petersen, R.C., Doody, R., Kurz, A., Mohs, R.C., Morris, J.C., Rabins, P.V., Ritchi, K., Rossor, M., Thal, L., \& Wingblad, B. (2001). Current concepts in mild cognitive impairment. Archives of Neurology, 58, 1985-1992.

Peterson, R.A. \& Heilbronner, R.L. (1987). The Anxiety Sensitivity Index: Construct validity and factor analytic structure. Journal of Anxiety Disorders, 1, 117-121.

Petersen, R.C. \& O’Brien, J. (2006). Mild cognitive impairment should be considered for DSM-V. Journal of Geriatric Psychiatry and Neurology, 19, 147-154.

Petersen, R.C., Smith, G.E., Waring, S.C., Ivnik, R.J., Tangalos, E.G., \& Kokmen, E. (1999). Mild cognitive impairment: Clinical characterization and outcome. Archives of Neurology, 56, 303-308.

Podewils, L.J., McLay, R.N., Rebok, G.W., \& Lyketsos, C.G. (2003). Relationship of self perceptions of memory and worry to objective measures of memory and cognition in the general population. Psychosomatics, 44, 461-470.

Rapp, S.R., Parisi, A., Walsh, D.A., \& Wallace, C.E. (1988). Detecting depression in elderly medical inpatients. Journal of Consulting and Clinical Psychology, 56, 509-513.

Reiss, S. (1991). Expectancy model of fear, anxiety, and panic. Clinical Psychology Review, 11, 141-153.

Reiss, S. \& McNally, R.J. (1985). Expectancy model of fear. In S. Reiss \& R.R. Bootzin (Eds.), Theoretical issues in behavior therapy (pp. 107-122). New York: Academic Press.

Reiss, S., Peterson, R.A., Gursky, D.M., \& McNally, R.J. (1986). Anxiety sensitivity, anxiety frequency, and the prediction of fearfulness. Behavior Research and Therapy, 24, 1-8.

Rey, A. (1964). L' examen clinique en psychologie. [The clinical examination in psychology]. Paris: Universitaires de France.

Saykin, A.J., Wishart, H.A., Rabin, L.A., Santulli, R.B., Flashman, L.A., West, J.D., McHugh, T.L., \& Mamourian, A.C. (2006). Older adults with cognitive complaints show brain atrophy similar to that of amnestic MCI. Neurology, 67, 834-842.

Schmand, B., Jonker, C., Hooijer, C., \& Lindeboom, J. (1996). Subjective memory complaints may announce dementia. $\mathrm{Neu}$ rology, 46, 121-125.

Schmidt, M. (1996). Rey Auditory and Verbal Learning Test: A Handbook. Los Angeles, CA: Western Psychological Service.

Schmidt, I.W., Berg, I.J., \& Deelman, B.G. (2001). Relations between subjective evaluations of memory and objective memory performance. Perceptual and Motor Skills, 93, 761-776.

Schmidt, N.B., Lerew, D.R., \& Joiner Jr., T.E. (1998). Anxiety sensitivity and the pathogenesis of anxiety and depression: Evi- dence for symptom specificity. Behaviour Research and Therapy, 36, 165-177.

Small, G.W., Chen, S.T., Komo, S., Ercoli, L., Bookheimer, S., Miller, K., Lavretsky, H., Saxena, S., Kaplan, A., Dorsey, D., Scott, W.K., Saunders, A.M., Haines, J.L., Roses, A.D., \& Pericak-Vance, M.A. (1999). Memory self-appraisal in middleaged and older adults with the Apolipoprotein E-4 allele. American Journal of Psychiatry, 156, 1035-1038.

Smith, G.E., Petersen, R.C., Ivnik, R.J., Malec, J.F., \& Tangalos, E.G. (1996). Subjective memory complaints, psychological distress, and longitudinal change in objective memory performance. Psychology and Aging, 11, 272-279.

SPSS Inc. (2005). SPSS for Windows (version 14) [Computer software]. Chicago, IL: Author.

Stegen, K., Van Diest, I., Van de Woestinje, K.P., \& Van den Bergh, O. (2001). Do persons with negative affective have an attentional bias to bodily sensations? Cognition \& Emotion, 15, $813-829$

Stewart, R., Russ, C., Richards, M., Brayne, C., Lovestone, S., \& Mann, A. (2001). Depression, APOE genotype and subjective memory impairment: A cross-sectional study in an African-Caribbean population. Psychological Medicine, 31, 431-440.

Sunderland, A., Watts, K., Baddeley, A.D., \& Harris, J.E. (1986). Subjective memory assessment and test performance in elderly adults. Journal of Gerontology, 41, 376-384.

Taylor, J.L., Miller, T.P., \& Tinklenberg, J.R. (1992). Correlates of memory decline: A 4-year longitudinal study of older adults with memory complaints. Psychology and Aging, 7, 185-193.

Telch, M.J., Lucas, J.A., \& Nelson, P. (1989). Nonclincal panic in college students: An investigation of prevalence and symptomatology. Journal of Abnormal Psychology, 98, 300-306.

Thomas, A.J., Kalaria, R.N., \& O'Brien, J.T. (2004). Depression and vascular disease: What is the relationship? Journal of Affective Disorders, 79, 81-95.

Turvey, C.L., Schultz, S., Arndt, S., Wallace, R.B., \& Herzog, R. (2000). Memory complaint in a community sample aged 70 and older. Journal of the American Geriatrics Society, 48, 1435-1441.

Wang, L., Belle, G.V., Crane, P.K., Kukull, W.A., Bowen, J.D., McCormick, W.C., \& Larson, E.B. (2004). Subjective memory deterioration and future dementia in people aged 65 and older. Journal of the American Geriatrics Society, 52, 2045-2051.

Watson, D. (1988). Intraindividual and interindividual analyses of positive and negative affect: Their relation to health complaints, percelived stress, and daily activities. Journal of Personality and Social Psychology, 54, 1020-1030.

Watson, D., Clark, L.A., \& Tellegen, A. (1988). Development and validation of brief measures of positive and negative affect: The PANAS scales. Journal of Personality and Social Psychology, 54, 1063-1070.

Watson, D. \& Pennebaker, J.W. (1989). Health complaints, stress and distress: Exploring the central role of negative affectivity. Psychological Review, 96, 234-254.

Yesavage, J.A., Brink, T.L., Rose, T.L., Lum, O., Huang, V., Adey, M., \& Leirer, V.O. (1983). Development and validation of a Geriatric Depression Scale. Journal of Psychiatric Research, 17, 37-49. 